\title{
Study protocol: evaluation of the addictive potential of e-cigarettes (EVAPE): neurobiological, sociological, and epidemiological perspectives
}

Sabine Vollstädt-Klein ${ }^{1,2^{*}}$ (], Nadja Grundinger ${ }^{1 \dagger}$, Tatiana Görig ${ }^{3}$, Daria Szafran ${ }^{3}$, Astrid Althaus ${ }^{4}$, Ute Mons ${ }^{4 \dagger}$ and Sven Schneider ${ }^{3+}$

\begin{abstract}
Background: Tobacco use is the largest preventable cause of diseases and deaths; reducing tobacco intake is, therefore, an urgent public health goal. In recent years, e-cigarettes have been marketed as a 'healthier' alternative to tobacco smoking, whilst product features have evolved tremendously in the meantime. A lively scientific debate has developed regarding the potential benefits and risks of e-cigarettes although, surprisingly, there are few studies investigating the addictive potential of nicotine-containing e-cigarettes. The present work comprises three work packages investigating the addictive potential of e-cigarettes from different perspectives: (1) the neurobiological addictive potential of e-cigarettes; (2) the experience and perception of dependence symptoms among users of e-cigarettes in a social context; and (3) the epidemiological perspective regarding factors influencing the potential for dependence.

Methods: Work package I: the neurobiological study will investigate the key elements of addiction in e-cigarettes compared to tobacco cigarettes using neurobiological and neuropsychological correlates associated with craving, incentive motivation, cue reactivity and attentional bias. Work package II: the sociological study part examines selfreports on the experience and perception of dependence symptoms in a social context, using focus group interviews and the analysis of posts in online discussion forums on e-cigarettes. Work package III: the epidemiological study part focuses on tolerance development and the role of psychosocial and product factors by analyzing longitudinal data from the International Tobacco Control Policy Evaluation Project (ITC).
\end{abstract}

Discussion: The present study offers a chosen mix of three methodological approaches, thereby comprehensively examining core symptoms of positive and negative reinforcement in addiction. Whether e-cigarettes are as reinforcing and addictive as combustible tobacco cigarettes is an important public health issue with implications for prevention and treatment programs.

Trial registration: Work package I: Registered at clinicaltrials.gov/ct2/show/NCT04772014. Work package II: Registered at OSF Registries: https://osf.io/dxgya (2021, January 14).

\footnotetext{
*Correspondence: sabine.vollstaedt-klein@zi-mannheim.de

†Sabine Vollstädt-Klein, Nadja Grundinger, Ute Mons and Sven Schneider

have contributed equally to this work

${ }^{1}$ Department of Addictive Behavior and Addiction Medicine, Central

Institute of Mental Health, Medical Faculty Mannheim, University

of Heidelberg, PO Box 1221 20,68072 Mannheim, Germany

Full list of author information is available at the end of the article
}

(C) The Author(s) 2021. Open Access This article is licensed under a Creative Commons Attribution 4.0 International License, which permits use, sharing, adaptation, distribution and reproduction in any medium or format, as long as you give appropriate credit to the original author(s) and the source, provide a link to the Creative Commons licence, and indicate if changes were made. The images or other third party material in this article are included in the article's Creative Commons licence, unless indicated otherwise in a credit line to the material. If material is not included in the article's Creative Commons licence and your intended use is not permitted by statutory regulation or exceeds the permitted use, you will need to obtain permission directly from the copyright holder. To view a copy of this licence, visit http://creativecommons.org/licenses/by/4.0/. The Creative Commons Public Domain Dedication waiver (http://creativeco mmons.org/publicdomain/zero/1.0/) applies to the data made available in this article, unless otherwise stated in a credit line to the data. 
Keywords: Electronic cigarettes, Addiction, Tobacco use disorder, Craving, Tolerance, fMRI, Focus groups, Longitudinal data

\section{Background}

Tobacco use causes more than 8 million deaths worldwide annually, making it the biggest preventable cause of disease [1]. Hence, reducing tobacco consumption and the associated health burden is an important goal. In 2006, e-cigarettes entered the market as alternatives to smoking tobacco cigarettes and the product characteristic have evolved tremendously since then. E-cigarettes can make nicotine available to users without exposing them to the harmful toxicants of tobacco smoke. Nevertheless, researchers have expressed different views on potential benefits and risks associated with e-cigarettes. Proponents see e-cigarettes as an innovative step in tobacco harm reduction, as switching from tobacco to e-cigarettes significantly reduces users' exposure to the main toxicants of tobacco smoke [2-6] and may help smokers quit tobacco use [7]. Opponents on the other hand point to the lack of long-term data regarding potential health risks $[8,9]$, and fear that the marketing of e-cigarettes as lifestyle products leads to nicotine dependence and might be a gateway or a catalyst to tobacco use, especially among young non-smokers [10-14]. Although this 'gateway hypothesis' is highly controversial [15], research indicates that e-cigarettesas compared to other nicotine replacement therapies (NRTs) - are mostly not limited to short-term use [16, 17]. Ex-smokers, who switch to e-cigarettes, often maintain their nicotine levels. While most e-cigarettes allow a gradual reduction of nicotine levels, longitudinal research findings indicates that a reduction in the concentration of nicotine may be accompanied by a higher consumption of liquid [18]. Such data suggests a maintenance of nicotine addiction that is initially acquired by tobacco use. It is however surprising that there are only few studies to date that examine the addictive potential of e-cigarettes containing nicotine.

Addictive behavior is determined by positive and negative reinforcement mechanisms. The rewarding potential of a substance includes its euphoric effect and the latency period until the effect occurs. The faster the drug enters the brain, the greater the euphoric effect $[19,20]$. This positive reinforcement effect leads to an initial repetition of drug use. Neuroadaptive changes occur, which lead to a dysregulation of the neurochemical circuits and thus to withdrawal symptoms when the drug is discontinued [21]. The shorter the elimination half-life of a substance, the more severe the withdrawal symptoms [20]. To avoid withdrawal symptoms, the substance is consumed repeatedly and frequently, leading to increasing tolerance (e.g., higher dosage to achieve the same effect) [19]. Accordingly, in addition to positive reinforcement in the early stages of the addiction process, this negative reinforcement mechanisms are increasingly recruited as a source of motivation [21].

Tobacco dependence is primarily produced by the pharmacological effects of nicotine [22]. Cigarette smoke releases significant amounts of nicotine into the bloodstream, where it quickly reaches the brain and triggers the release of dopamine by stimulating nicotinic acetylcholine receptors [23]. Other characteristics and additives of tobacco smoke further enhance the addictive potential [24-27]. This causes the rapid positive reinforcement, making smoking of tobacco the most addictive form of nicotine administration [22, 28]. Nicotine uptake is significantly slower and lower with NRTs, which can explain the absence of the addiction-inducing 'kick' [29]. In most studies, e-cigarettes also showed lower nicotine absorption than tobacco cigarettes [30]. If they deliver nicotine less effectively, e-cigarettes might, thus, have less addictive properties. Nevertheless, depending on the device, liquid, and user behavior, it is possible to achieve equal or even higher plasma nicotine levels [30, 31].

However, nicotine is a necessary but not a sufficient component in the development of dependence [32]. Non-pharmacological motives for smoking include psychological, behavioral, sensorimotor and social manipulative factors [33, 34]. Thus, cigarette smoke is known to have very characteristic sensory effects on the respiratory tract that are perceived as pleasant and reduce the urge to smoke even more effectively than the direct pharmacological effect of nicotine [32, 35-37]. The combination of pleasant stimuli associated with smoking behavior and the drug itself act synergistically. Multisensory experiences of smoking (visual, tactile, auditory, olfactory, gustatory) quickly acquire the quality of a conditioned cue stimulus that can trigger the urge to smoke [38]. Therefore, craving can be induced by the substance itself, substance-associated stimuli or by emotional states such as stress $[39,40]$. This is not necessarily associated with physical discomfort, but also includes preoccupation with thoughts of the drug. Expectations of positive outcomes from smoking (e.g., social interaction, stress coping, stop craving) as well as expectations of the negative consequences of quitting (e.g., physical withdrawal symptoms, weight gain) play a crucial role in addiction. Thus, it has been shown that $79 \%$ of interviewed ex-smokers 
are afraid of relapsing if they would stop using their e-cigarette [41]. Therefore, psychological dependence is also characterized by repeated drug use, but this is based less on tolerance development or physical withdrawal symptoms and more on classical and operant conditioning processes and craving [19]. With repeated consumption, the initial hedonic effects finally diminish, while consumption increasingly becomes habitual and eventually compulsive [42]. Cigarette smoking is such a compulsive behavioral pattern: rigid, automatic, and habitual actions that require little mental elaboration and are triggered by internal or external stimuli. E-cigarettes are the only tobacco-free nicotine delivery devices that closely resemble the smoking ritual of cigarette smoking: Handto-mouth movement, tactile action of puffing, inhalation and exhalation, the sensory stimulus in the airways, vapor production and social aspects such as smoking breaks. Therefore, e-cigarettes might be expected to produce the same psychological, behavioral, and social effects that can promote or maintain nicotine dependence.

Epidemiological data suggest that e-cigarettes may lead to dependence symptoms, such as craving, or e-cigarette use within $30 \mathrm{~min}$ of waking. In these studies, the severity of dependence, however, was significantly lower with e-cigarettes compared to tobacco cigarettes [43-45]. This is consistent with self-reporting by users, many of whom state that they are less dependent upon their e-cigarette than they were previously upon tobacco cigarettes [17, 46, 47]. Some experimental studies show that e-cigarettes, compared to other 'high- and low-abuse liability'-nicotine products, have some risk of abuse that appears to be higher than for NRTs but lower compared to tobacco cigarettes [48-50]. Nevertheless, e-cigarette users show greater discounting for liquid compared to money, which was associated with more unsuccessful attempts to quit vaping [51].

Research to date shows that e-cigarettes have the potential for abuse liability and to maintain an existing nicotine dependence and lead to dependence symptoms. Whether e-cigarettes have a similar addictive potential as tobacco cigarettes has not yet been sufficiently clarified, especially since many studies were still conducted on old devices from earlier generations. Newer, more powerful devices can deliver nicotine more efficiently. It is still unclear what role dependence symptoms such as craving, tolerance and withdrawal symptoms play in e-cigarette use and how they develop.

This research hence comprises three work packages with the aim of investigating the addictive potential of e-cigarettes from three different perspectives, combining neurobiological, sociological, and epidemiological research methods and levels of observation. In particular, craving is examined as a correlate for reward potential and tolerance development as a correlate for punishment potential. By combining these complementary methodological approaches, the overall project aims to cover all relevant sub-constructs of the addictive potential of e-cigarettes.

\section{Work package I: neurobiological study part}

Registered at clinicaltrials.gov/ct2/show/NCT04772014.

\section{Objectives}

In this work package (WP), the focus is on the investigation of positive reinforcement mechanisms of e-cigarettes utilizing neurobiological and neuropsychological methods. One of the most discussed theories in this context is the incentive sensitization theory by Berridge and Robinson: Accordingly, mesolimbic sensitization occurs with repeated drug use, leading to a realignment of the reward and motivation system, resulting in the attribution of incentive salience of drug-associated stimuli, making them attractive and 'wanted' [52-54]. Thus, the substance and its stimuli are attributed a high reward value, which can be measured in terms of effort, time, money, or other goods one is willing to spend to acquire it. Some experimental studies on tobacco smokers $[50,55]$ and experienced dual users [56] show that tobacco cigarettes have a higher reward value than e-cigarettes. However, most participants smoked more frequently and for a much longer period of time, which is why the reward value for tobacco cigarettes could be more established. Thus, the reward value of cigarettes itself was found to differ between dependent and occasional smokers. Occasional smokers exert more physical effort to obtain money and showed increased reactivity of the mesocorticolimbic system (including ventral striatum) to stimuli that predicted a money reward compared with a cigarette reward. Dependent smokers, in contrast, exerted similar physical effort and showed equivalent anticipatory activity for both reward types [57]. Measuring brain activity in a heterogeneous e-cigarette consumer group during reward announcement and acquisition for tobacco cigarettes and e-cigarettes could provide additional information about their reward value.

In addition, numerous meta-analyses in tobacco smokers show that smoking-related cues elicit significantly greater craving in smokers than neutral cue stimuli. This is associated with distinctive neural activation patterns in, e.g., the striatum, amygdala, orbitofrontal cortex, anterior cingulate cortex, medial prefrontal cortex and insula [58-61]. There are few studies on cue reactivity with e-cigarettes and they deliver conflicting results. In a study with merely seven participants, Nichols and colleagues failed to detect e-cigarette cue-related activity in brain areas associated with cue reactivity; but in regions 
related to episodic memory retrieval and motor control [62]. Another study by Wall and colleagues examined 10 subjects using e-cigarettes during functional magnetic resonance imaging (fMRI) to visualize brain activity associated with active vaping. Activation clusters were seen in cortical regions, (e.g., the insula, amygdala, and the anterior cingulate gyrus) as well as in sub-cortical regions (e.g., in the thalamus and putamen). Relative deactivations associated with vaping were detected in parts of the ventral striatum [63]. One possible explanation may be the transition from goal-directed to habitual behavior in e-cigarette use that has been associated with a dysfunction of fronto-striatal circuits and a shift from ventral to dorsal striatal responses [64].

Furthermore, cognitive processes play an essential role in reactivity to drug cues. Through mesolimbic sensitization drug stimuli automatically and involuntary become the focus of attention. Attentional bias has been consistently found in various substance use disorders [65] and also in smokers [66-69]. Studies have shown that smokers have an initial orientation to smoking-related cues [70] and maintain their gaze on smoking-related images longer than on control images $[67,71]$. In fact, current tobacco smokers also have a longer dwell time on e-cigarette cues compared to neutral cues, which was associated with greater baseline craving [72]. However, to our best knowledge, there is no study investigating attentional bias for tobacco cigarette and e-cigarette stimuli in e-cigarette users.

In sum, the neuropsychological and neurobiological mechanisms of cigarette dependence have been relatively well studied. The extent to which this can be applied to e-cigarettes has not yet been adequately elucidated. Therefore, the neurobiological study will investigate the key elements of addiction in e-cigarettes compared to tobacco cigarettes using neurobiological and neuropsychological correlates associated with craving, incentive motivation, cue reactivity and attentional bias. We hypothesize that (1) participants who mainly use e-cigarettes work harder for e-cigarettes and show increased activation in the ventral striatum in the anticipation phase for e-cigarettes compared to tobacco cigarettes; (2) e-cigarette users show increased cue reactivity (compared to nicotine naïve users) in response to e-cigarette stimuli compared to neutral stimuli. Moreover, dual users will show activations in the same neural networks for tobacco cigarette and e-cigarette stimuli; (3) e-cigarette users (compared to nicotine naïve and dual users) show an increased attentional bias towards e-cigarette cues, which correlates positively with e-cigarette use. Dual users' attentional bias towards smoking cues, on the other hand, correlates positively with tobacco cigarette use.

\section{Methods Study sample}

We intend to include 70 e-cigarette users (daily e-cigarette use, additional smoking of tobacco cigarettes is not an exclusion criterion) and 30 nicotine naïves (lifetime consumption of less than 20 e-cigarettes or tobacco cigarettes) aged 18-65 years. Exclusion criteria for both groups are contraindications for an MRI examination, severe internal, neurological, and psychiatric comorbidities, pharmacotherapy with psychoactive substances within the past 14 days, current substance abuse (THC, amphetamine, opiates, benzodiazepines, barbiturates, and cocaine) and axis I disorders according to ICD-10 and DSM-5 (except tobacco use disorder and specific phobias).

\section{Power calculation}

Sample size was estimated with an assumed effect size of $\rho=0.3$ using G*Power software tool version 3 [73]. In this case, $\mathrm{n}=64$ smokers would be sufficient to detect a correlative relationship at $p<0.05$ with a power of $80 \%$. Since dropouts due to artifacts or lack of compliance are to be expected, a total of 70 subjects will be examined. The number of cases in the healthy control group $(n=30)$ is also sufficient to detect group differences between smokers and nicotine naïve subjects (effect size $\mathrm{d}=0.6 ; 80 \%$ power, $p<0.05)$.

\section{Study design}

Inclusion and exclusion criteria are checked in advance in a telephone interview. The subjects are comprehensively informed about the objectives and the procedure of the planned examinations. On the examination day, smoking status is checked by measuring carbon monoxide levels in exhaled air and by taking a saliva sample to determine cotinine levels. Sociodemographic data are collected. Drug urine screening is performed, as well as a pregnancy test for women. This is followed by diagnostic interviews and the recording of smoking and vaping behavior, the severity of dependence symptoms, craving, and expected consequences for the use of tobacco cigarettes and e-cigarettes, as well as withdrawal symptoms using standardized questionnaires. Psychiatric and neurological, as well as somatic pre-existing conditions are recorded, as is the subject's current medication. In a neuropsychological assessment, subjects complete an Implicit Association Task, Delay Discounting Task, and Iowa Gambling Task. The fMRI examination is performed with a $3 \mathrm{~T}$ whole-body tomograph (Siemens Healthineers, Erlangen, Germany) including restingstate, MPRAGE and three different tasks: (1) To measure the reward value of e-cigarettes, we use an instrumental motivation task (adapted from [57]). Thereby, we 
examine brain activity to reward-predicting stimuli (cigarette, liquid, and money) and the subsequent instrumental response to obtain the reward. Physical effort (pressing a button) is thus used as a measure of motivation. (2) In a cue reactivity paradigm (adapted from [74]) participants' physiological and neural responses, as well as self-reported craving, are examined while viewing images of e-cigarettes and tobacco cigarettes compared to neutral pictures. (3) Attentional bias for e-cigarette and tobacco cigarette cues and its neural correlates are tested using a visual dot-probe task (adapted from [69]). One problem is that the reaction time-based index only provides a 'snapshot' of attention, which can be overcome by directly measuring participants' eye movements during the task. To improve reliability, this task is, therefore, combined with eye tracking (see also $[75,76]$ ). For a comprehensive list of questionnaires and tasks used, please see Table 1. For a graphical representation of the paradigms used during fMRI, please see Fig. 1.

\section{Work package II: sociological study part}

Registered at OSF Registries: https://osf.io/dxgya.

\section{Objectives}

In this part of the study program, the question is whether the reward potential (e.g., craving) and the punishment potential (e.g., tolerance development) are actually subjectively perceived by e-cigarette users. Therefore, the aim of the sociological study part (WP II) is to investigate whether aspects of addiction defined in the current DSM-5 are also reported by the users themselves. To this end, two different qualitative approaches will be combined.

Table 1 Measurements implemented in WP I

Initial information

Nicotine consumption

Emotional state

Neuropsychological assessment

fMRI examination

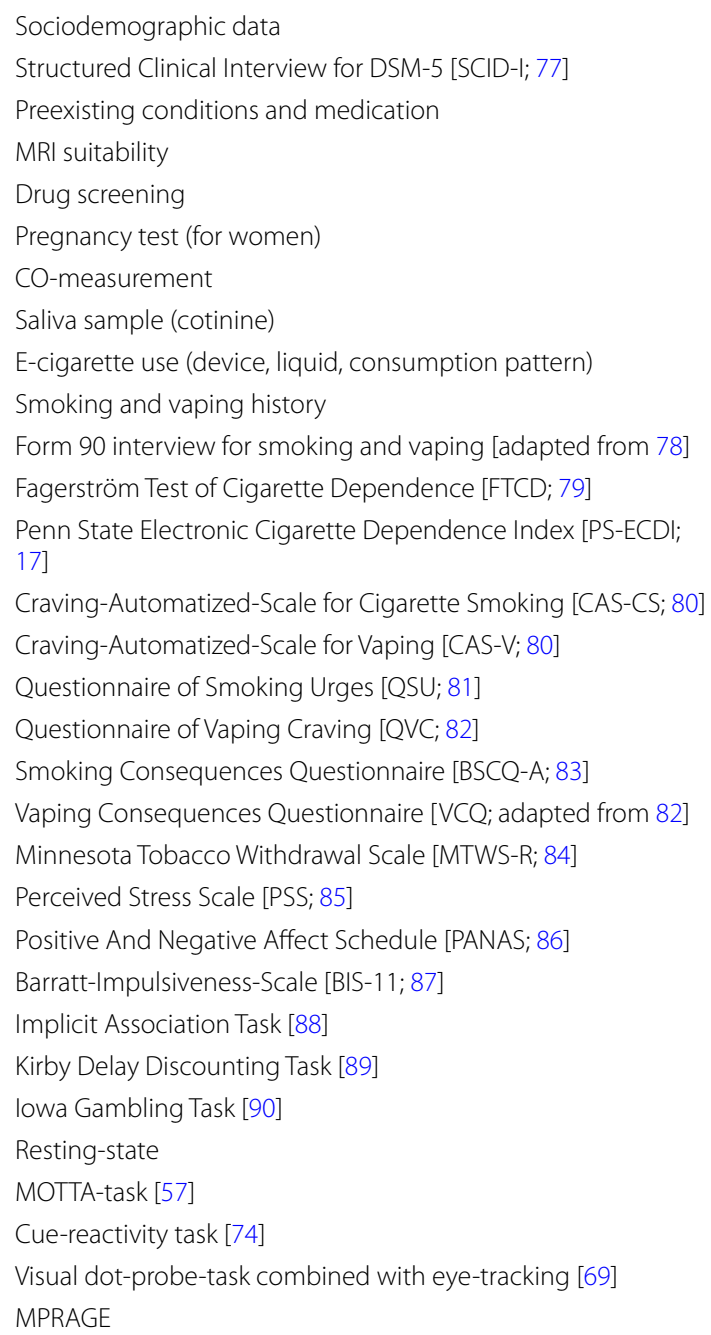


Instrumental Motivation Task

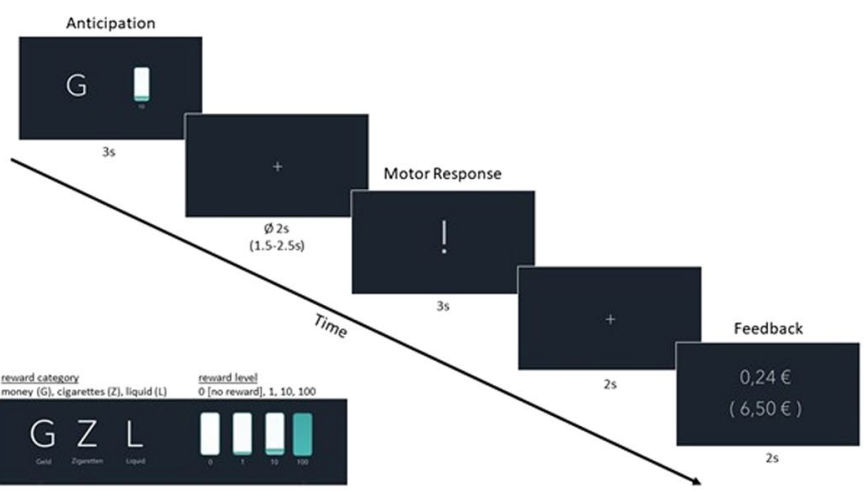

Cue-Reactivity

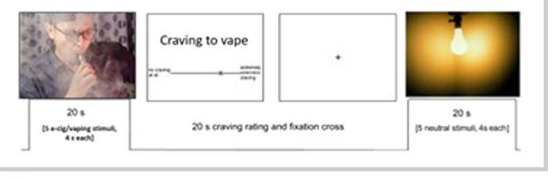

Visual Dot-Probe-Task

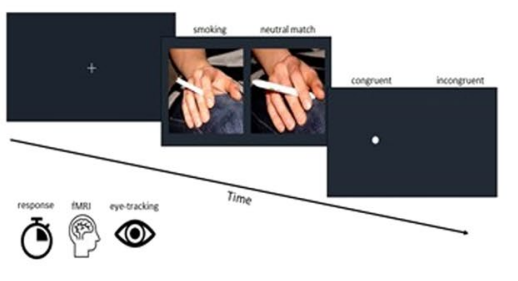

Fig. 1 Experimental tasks used during fMRI. Note. (1) Instrumental motivation task (adapted from [57]); (2) Cue-reactivity paradigm (adapted from [74]); (3) visual dot-probe task (adapted from [69])

\section{Methods}

\section{Focus group interviews}

One approach within the sociological study part (WP II) is to conduct four focus group interviews, each with 9-10 e-cigarette users. The planned focus group interviews aim to capture potential experiences of craving and tolerance development. An open-ended guide will be developed for conducting the focus group interviews. Inclusion criteria for participants will be: (1) age $\geq 18$ years, (2) sufficient understanding of the German language, and (3) daily e-cigarette use. Dual use of tobacco and e-cigarettes will be set as a specific exclusion criterion for participation in this study part to exclude dependence symptoms resulting from the tobacco cigarette use. The composition of the focus groups will be as heterogeneous as possible [91] in order to generate a wide variety of opinions and to discuss as many different experiences as possible.

Prior to the focus group interview, each participant will be personally informed about the objectives of the study and data protection procedures. A written consent will be obtained from each participant. After completion of each focus group (max. $2 \mathrm{~h}$ ), all participants will receive information on the current state of research on health risks and dependence potential of e-cigarettes. In addition, participants will receive an expense allowance of $50 €$ and the opportunity to receive information about the study results at a later date. All focus group discussions will be audiotaped, transcribed verbatim, and analyzed using qualitative content analysis [92].

\section{Online forums}

In addition to the focus group interviews, an analysis of posts in online discussion forums on e-cigarettes will be conducted. Online forums represent anonymous places of exchange for users [93] and an opportunity to share ideas about problematic or taboo topics without fear of stigmatization [94]. We hypothesize that the internet, and in particular anonymous online forums, are among the few places where e-cigarette users report possible dependence symptoms without feeling shame. Therefore, they provide a venue to gain health- and dependencerelated experiences without the social-desirability bias [95]. Examining posts in online forums complements focus group interviews in an innovative and useful way, as shame-related experiences may be reported more detailed than in focus group interviews.

A three-step procedure will be used to collect the data for this study part. In the first step, the relevant online forums will be identified via Google search using different combinations with various spellings of the German words "e-cigarette" and "(online) forum". Following inclusion criteria will be applied to select relevant online forums: (1) e-cigarettes as the main topic of the forum, (2) the forum is in German language; (3) the forum is publicly accessible (i.e., no registration is required to read the users contributions, (4) the forum was active over the previous 4 weeks, (5) at least 500,000 posts, (6) at least 5000 forum members, (7) search function within the forum, (8) no affiliation with tobacco industry, (9) public disclaimer in terms and conditions. In the second step, the identified forums will be searched for previously 
defined keywords describing dependence criteria derived from current DSM-5 covering the reward potential (e.g., craving) and the punishment potential (e.g., tolerance development) as accurate as possible. In the third step, the identified user contributions will be analyzed using qualitative content analysis in regard to the reported dependence symptoms.

As suggested in a previous discussion about ethics of using of online data [96], formal ethical clearance is not necessary for analyses of such kind of posts in online discussion forums. We will use data that is publicly accessible at the time of data collection, so that forum members can be assumed to be aware of the public availability of their posts. Nicknames of users will not be included in data analyses, and no further information on individuals is available in the forums. The team members will not participate actively in any discussions in the forums.

\section{Work package III: epidemiological study part Objectives}

The aim of WP III is to quantify dependence symptoms, in particular the development of tolerance, in e-cigarette users and to investigate associations with user and product factors within the framework of a secondary data analysis of a representative large-scale longitudinal study of tobacco and e-cigarette use. In particular, we will investigate how dependence symptoms develop over time. For this purpose, transitions to e-cigarette use or from tobacco cigarette use will be investigated. Associations of such transitions with individual factors (e.g., age, gender, socioeconomic factors) and attitudes and perceptions (e.g., perceived dependence and harm potential of tobacco and e-cigarettes, perceived societal norms regarding tobacco and e-cigarette use) will be studied. In addition, because most e-cigarette users were previously long-time smokers of conventional tobacco cigarettes or continued to smoke tobacco cigarettes, the comparison of the perceived addictive potential of both products is of interest.

\section{Methods}

WP III mainly includes a secondary data analysis of already collected and available longitudinal data from the International Tobacco Control Policy Evaluation Project (ITC) - a multinational consortium comprising longitudinal surveys on representative samples of smokers using largely standardized survey instruments and methods [97]. Conceptually, the survey instruments and models of the ITC cigarette project are based on psychosocial behavioral theories [98]. For the planned analyses, two survey waves are used, which were collected in six European countries (Germany, Greece, Poland, Romania, Spain, Hungary) within the EU-funded project
EUREST-PLUS [99]. The baseline survey took place from June to September 2016 and included approximately 1000 smokers per country (total: $\mathrm{N}=6011$ ). Participants were recruited using multistage cluster sampling, geographically stratified by Nomenclature of Territorial Units for Statistics-Region (NUTS) region. A random walk procedure was used to randomly select addresses of households from 100 clusters in each country. Households were eligible if at least one smoker $(>100$ cigarettes smoked in lifetime and at least monthly cigarette consumption) lived there. A maximum of one male and one female smoker from each selected household were randomly selected for a computer-assisted interview [100]. The second wave of the survey took place between February and May 2018, during which participants from the first wave of the survey were interviewed a second time. Overall, $54 \%$ of participants from the first wave participated a second time. For participants who refused to participate a second time or could not be reached (socalled panel mortality), replacement participants were recruited analogous to the initial sample selection to enable cross-sectional analyses in addition to longitudinal analyses [101]. This database is supplemented by two further surveys of the ITC cigarette Europe project (the Netherlands: approx. $\mathrm{N}=2000$ smokers, and England: approx. $N=4300$ smokers, former smokers, and vapers), which were not collected within the scope of the EUREST-PLUS project, but which have good comparability given the use of the ITC cigarette sampling design and data collection methods across all involved countries [101]. All study participants provided informed consent and all study procedures and material were approved by the ethics research committee at the University of Waterloo (Ontario, Canada), and local ethics committees in all countries.

Of relevance for this work package are dependence symptoms, which were recorded with identical question wording at both survey time points and separately for tobacco cigarettes and e-cigarettes, depending on which products are being used. Pertinent measures include, in particular, self-reports (e.g., time of first use of cigarettes or e-cigarettes after getting up in the morning, failed attempts at abstinence, need for daily functioning) and self-assessments (e.g., of the degree of dependence). Furthermore, measures of perceived addictiveness of products are available. Tolerance development can be mapped longitudinally via detailed recording of dose for both tobacco cigarettes and e-cigarettes.

Measures of interest and associations with individual and product factors will be studied cross-sectionally using regression models. To examine the course of dependence symptoms over time based on longitudinal data, generalized linear models are the method of choice 
to account for intra-individual correlation. In order to investigate to what extent trajectories or transitions are influenced by individual and product characteristics, these are introduced into the models as influencing factors (i.e., modeled as interaction terms with the time factor).

\section{Discussion}

The addictive potential of tobacco cigarettes is undisputed. E-cigarettes are very similar to tobacco cigarettes in their nicotine delivery and smoking behavior: Handto-mouth movement, tactile action of puffing, inhalation and exhalation, sensory stimulation in the airways, nicotine uptake via the pulmonary route, vapor production and social aspects such as smoking breaks. Therefore, e-cigarettes could produce the same pharmacological, psychological, behavioral, and social effects that can promote or maintain nicotine dependence. However, there are few studies on the addictive potential of e-cigarettes containing nicotine-with conflicting results. With the present project, we aim to close this gap by investigating the addictive potential of e-cigarettes from three perspectives, combining different research methods and levels of observation:

(1) The neurobiological study part focuses on the positive reinforcement mechanisms of e-cigarettes using neurological and neuropsychological research methods. On the neurobiological level, the reward value of e-cigarettes and craving will be investigated in an experimental approach through presentation of conditioned stimuli and measurement of motivational and attentional processes. The aim is to test the assumption that chronic use of e-cigarettes leads to similar conditioning processes and motivational aspects as with traditional tobacco cigarettes.

(2) The sociological study part uses a qualitative approach to investigate the extent to which e-cigarette users actually experience and report craving and tolerance development. This involves a qualitative description of the typical experience and perception of dependence symptoms in a social context. The self-reports of users cover psychological, physiological, and behavioral aspects of dependence disorders.

(3) The epidemiological study part examines the factors influencing the potential for dependence and the development of dependence symptoms in a longitudinal study. The focus is on the development of tolerance and the role played by psychosocial and product factors with regard to transitions into and out of e-cigarette use. Using readily available quantitative longitudinal data, the development of toler- ance among e-cigarette users will be quantified and associations with user and product factors examined.

During the conduct of this project, we will have to deal with certain limitations. Most e-cigarette users are former or current smokers. Here, the dependence symptoms or the dependent behavior could reflect the transfer of nicotine dependence from the previous use of combustible tobacco. Thus, it cannot be clarified whether the use of e-cigarettes alone actually leads to the development of addiction. Ideally, an evaluation of the addictive potential would be done with a group of individuals using nicotinecontaining e-cigarettes but who have never used tobacco products. However, the prevalence of exclusive e-cigarette users who have never smoked cigarettes in their life is very low. Furthermore, previous or concurrent cigarette use also plays a role in the examination of reward effects such as craving and incentive motivation. Thus, most users have a longer smoking history and may have a greater sensitivity to tobacco cigarette and smoking stimuli. Therefore, we collect extensive smoking variables (age of smoking initiation, duration of smoking, exposure to tobacco cigarettes measured by pack years, severity of dependence on tobacco cigarette) to statistically account for these potential confounders. From a methodological point of view, the heterogeneous product group of e-cigarettes and different liquids and nicotine concentrations must also be taken into account. This can make it difficult to analyze and compare the data collected. Therefore, extensive information about e-cigarette consumption is collected (e-cigarette device, coil model, nicotine concentration, flavor etc.). Nevertheless, e-cigarettes differ not only in their characteristics, nicotine delivery and consumption patterns, but also in their design, which makes it difficult to select suitable stimuli. This is problematic given that personalized, familiar stimuli can best trigger craving and attention biases [75].

The strength of the present study is the chosen mix of three methodological approaches, whereby core symptoms of positive and negative reinforcement in addiction are investigated comprehensively. The reward effects of e-cigarette and craving can be experimentally validated at the individual level, while longitudinal designs are the method of choice for measuring tolerance development as a correlate of the punishment potential. The simultaneous individual occurrence of both phenomena can furthermore be investigated through qualitative analysis of self-reports.

Whether e-cigarettes are as reinforcing and addictive as combustible tobacco cigarettes is an important public health question with implications for prevention and treatment programs. In particular, the development of 
tolerance towards e-cigarettes is relevant from a public health perspective, as health risks are usually higher with increasing consumption. In contrast, a systematic assessment of the reward value of e-cigarettes, especially in comparison to tobacco cigarettes, plays an important role for therapy offers. The results will provide important insights into the motivational properties of e-cigarettes and could expand our understanding of whether and to what extent e-cigarettes can be used in smoking cessation treatments.

\author{
Abbreviations \\ fMRI: Functional magnetic resonance imaging; ITC: International tobacco \\ control policy evaluation project; NRT: Nicotine replacement therapy; WP: \\ Work package.
}

\section{Acknowledgements}

The authors would like to thank Falk Kiefer for providing the infrastructure and support to conduct the study and Ronald Fischer for technical support during the study set-up.

\section{Authors' contributions}

SV, UM, and SS were responsible for the study design and procured study funding. SV, NG, UM, and SS drafted the manuscript with extensive input from $\mathrm{TG}, \mathrm{AA}$, DS. All authors made substantial contributions to the subsequent draft of the manuscript. All authors read and approved the final manuscript.

\section{Funding}

Open Access funding enabled and organized by Projekt DEAL. The project described in this study protocol is funded with a grant from the Deutsche Forschungsgemeinschaft (DFG, German Research Foundation), Project-ID 437718741. It is partly supported by another grant from the DFG, Project-ID 402170461 (recipient SVK, Heinz et al., Addict Biol. 2019). UM is supported by the Marga and Walter Boll-Foundation. The sources of funding have no role in study design, data collection, data analysis, data interpretation, or writing of the manuscript.

\section{Availability of data and materials}

Not applicable.

\section{Declarations}

\section{Ethics approval and consent to participate}

WP I was approved by the Ethics Committee II of the Medical Faculty Mannheim at the University of Heidelberg, Germany (Ethics approval number: 2018-595 N with amendment from 2021-01-13) and conforms to the requirements of the World Medical Association's Declaration of Helsinki. All procedures of WP II were approved by the Ethics Committee II of the Medical Faculty Mannheim at the University of Heidelberg (Ethics approval number: 2017-567 N-MA with amendment from 2019-07-15). Study procedures and material used by WP III was approved by the ethics research committee at the University of Waterloo (Ontario, Canada), and local ethics committees in all countries where surveys took place. For WP I and II, written informed consent is obtained from all participants. For WP III, informed consent was obtained from other research institutions where the surveys took place.

\section{Consent for publication}

Not applicable.

\section{Competing interests}

The authors declare that they have no competing interests.

\section{Author details}

'Department of Addictive Behavior and Addiction Medicine, Central Institute of Mental Health, Medical Faculty Mannheim, University of Heidelberg, PO
Box 1221 20, 68072 Mannheim, Germany. ${ }^{2}$ Mannheim Center for Translational Neurosciences (MCTN), Medical Faculty of Mannheim, University of Heidelberg, Mannheim, Germany. ${ }^{3}$ Mannheim Institute of Public Health, Social and Preventive Medicine (MIPH), Medical Faculty of Mannheim, University of Heidelberg, Mannheim, Germany. ${ }^{4}$ Heart Center, Faculty of Medicine, University Hospital Cologne, University of Cologne, Cologne, Germany.

Received: 29 September 2021 Accepted: 5 November 2021

Published online: 18 November 2021

\section{References}

1. World Health Organization. WHO report on the global tobacco epidemic 2019: offer help to quit tobacco use. Geneva: World Health Organization; 2019

2. Goniewicz ML, Knysak J, Gawron M, Kosmider L, Sobczak A, Kurek J, Prokopowicz A, Jablonska-Czapla M, Rosik-Dulewska C, Havel C, et al. Levels of selected carcinogens and toxicants in vapour from electronic cigarettes. Tob Control. 2014;23(2):133-9.

3. Hajek P, Etter JF, Benowitz N, Eissenberg T, McRobbie H. Electronic cigarettes: review of use, content, safety, effects on smokers and potential for harm and benefit. Addiction (Abingdon, England). 2014;109(11):1801-10.

4. Levy DT, Borland R, Lindblom EN, Goniewicz ML, Meza R, Holford TR, Yuan Z, Luo Y, O'Connor RJ, Niaura R, et al. Potential deaths averted in USA by replacing cigarettes with e-cigarettes. Tob Control Int J. 2018:27(1):18-25.

5. McNeill A, Brose LS, Calder R, Hitchman SC, Hajek P, McRobbie H. E-cigarettes: the need for clear communication on relative risks. Lancet. 2015;386(10000):1237

6. National Academies of Sciences, Engineering, and Medicine. Public health consequences of e-cigarettes. Washington: The National Academies Press; 2018.

7. Hartmann-Boyce J, McRobbie H, Lindson N, Bullen C, Begh R, Theodoulou A, Notley C, Rigotti NA, Turner T, Butler AR, et al. Electronic cigarettes for smoking cessation. Cochrane Database Syst Rev. 2021;4(4):Cd010216.

8. Hiemstra PS, Bals R. Basic science of electronic cigarettes: assessment in cell culture and in vivo models. Respir Res. 2016;17(1):127.

9. Pisinger C, Døssing M. A systematic review of health effects of electronic cigarettes. Prev Med. 2014;69:248-60.

10. Barrington-Trimis JL, Urman R, Berhane K, Unger JB, Cruz TB, Pentz MA, Samet JM, Leventhal AM, McConnell R. E-cigarettes and future cigarette use. Pediatrics. 2016;138(1):e20160379.

11. Loukas A, Marti CN, Cooper M, Pasch KE, Perry CL. Exclusive e-cigarette use predicts cigarette initiation among college students. Addict Behav. 2018:76:343-7.

12. Morgenstern M, Nies A, Goecke M, Hanewinkel R. E-cigarettes and the use of conventional cigarettes. Deutsch Ärzteblatt Int. 2018:115(14):243-8.

13. Pierce JP, Chen R, Leas EC, White MM, Kealey S, Stone MD, Benmarhnia T, Trinidad DR, Strong DR, Messer K. Use of E-cigarettes and other tobacco products and progression to daily cigarette smoking. Pediatrics. 2021;147(2):e2020025122

14. Schneider S, Diehl K. Vaping as a catalyst for smoking? An initial model on the initiation of electronic cigarette use and the transition to tobacco smoking among adolescents. Nicotine Tob Res Off J Soc Res Nicotine Tob. 2016;18(5):647-53.

15. Chan GCK, Stjepanović D, Lim C, Sun T, Shanmuga Anandan A, Connor JP, Gartner C, Hall WD, Leung J. Gateway or common liability? A systematic review and meta-analysis of studies of adolescent e-cigarette use and future smoking initiation. Addiction (Abingdon, England). 2021;116(4):743-56.

16. Hajek P, Phillips-Waller A, Przulj D, Pesola F, Myers Smith K, Bisal $\mathrm{N}$, Li J, Parrott S, Sasieni P, Dawkins L, et al. A randomized trial of e-cigarettes versus nicotine-replacement therapy. N Engl J Med. 2019:380(7):629-37.

17. Foulds J, Veldheer S, Yingst J, Hrabovsky S, Wilson SJ, Nichols TT, Eissenberg T. Development of a questionnaire for assessing dependence on electronic cigarettes among a large sample of ex-smoking 
E-cigarette users. Nicotine Tob Res Off J Soc Res Nicotine Tob. 2015;17(2):186-92.

18. Etter JF. A longitudinal study of cotinine in long-term daily users of e-cigarettes. Drug Alcohol Depend. 2016;160:218-21.

19. Nutt D, King LA, Saulsbury W, Blakemore C. Development of a rational scale to assess the harm of drugs of potential misuse. Lancet. 2007:369(9566):1047-53.

20. Salerian A. Addictive potency of substances. Pharm Pharmacol Int J. 2015. https://doi.org/10.15406/ppii.2015.02.00030.

21. Koob GF. Neurobiological substrates for the dark side of compulsivity in addiction. Neuropharmacology. 2009;56(Suppl 1):18-31.

22. Benowitz NL, Hukkanen J, Jacob P 3rd. Nicotine chemistry, metabolism, kinetics and biomarkers. Handb Exp Pharmacol. 2009;192:29-60.

23. Hukkanen J, Jacob P, Benowitz NL. Metabolism and disposition kinetics of nicotine. Pharmacol Rev. 2005;57(1):79-115.

24. Fowler JS, Logan J, Wang GJ, Volkow ND. Monoamine oxidase and cigarette smoking. Neurotoxicology. 2003;24(1):75-82.

25. Guillem K, Vouillac C, Azar MR, Parsons LH, Koob GF, Cador M, Stinus L. Monoamine oxidase inhibition dramatically increases the motivation to self-administer nicotine in rats. J Neurosci Off J Soc Neurosci. 2005;25(38):8593-600.

26. Lewis A, Miller JH, Lea RA. Monoamine oxidase and tobacco dependence. Neurotoxicology. 2007;28:182-95.

27. Stevenson T, Proctor RN. The secret and soul of Marlboro: Phillip Morris and the origins, spread, and denial of nicotine freebasing. Am J Public Health. 2008;98(7):1 184-94.

28. Henningfield JE, Keenan RM. Nicotine delivery kinetics and abuse liability. J Consult Clin Psychol. 1993;61 (5):743-50.

29. West R, Hajek P, Foulds J, Nilsson F, May S, Meadows A. A comparison of the abuse liability and dependence potential of nicotine patch, gum, spray and inhaler. Psychopharmacology. 2000;149(3):198-202.

30. Jacobson K, Martinez J, Larroque S, Jones IW, Paschke T. Nicotine pharmacokinetics of electronic cigarettes: a pooled data analysis from the literature. Toxicol Rep. 2021;8:84-95.

31. Breland A, Soule E, Lopez A, Ramôa C, El-Hellani A, Eissenberg T. Electronic cigarettes: What are they and what do they do? Ann N Y Acad Sci. 2017;1394(1):5-30.

32. Rose JE. Nicotine and nonnicotine factors in cigarette addiction. Psychopharmacology. 2006;184(3):274-85.

33. Buchhalter AR, Acosta MC, Evans SE, Breland AB, Eissenberg T. Tobacco abstinence symptom suppression: the role played by the smoking-related stimuli that are delivered by denicotinized cigarettes. Addiction (Abingdon, England). 2005;100(4):550-9.

34. Tate JC, Pomerleau CS, Pomerleau OF. Pharmacological and nonpharmacological smoking motives: a replication and extension. Addiction. 1994;89(3):321-30.

35. Barrett SP. The effects of nicotine, denicotinized tobacco, and nicotine-containing tobacco on cigarette craving, withdrawal, and self-administration in male and female smokers. Behav Pharmacol. 2010;21(2):144-52.

36. Naqvi $\mathrm{NH}$, Bechara A. The airway sensory impact of nicotine contributes to the conditioned reinforcing effects of individual puffs from cigarettes. Pharmacol Biochem Behav. 2005:81 (4):821-9.

37. Rose JE, Behm FM, Westman EC, Johnson M. Dissociating nicotine and nonnicotine components of cigarette smoking. Pharmacol Biochem Behav. 2000;67(1):71-81.

38. Niaura R, Shadel WG, Abrams DB, Monti PM, Rohsenow DJ, Sirota A. Individual differences in cue reactivity among smokers trying to quit: effects of gender and cue type. Addict Behav. 1998;23(2):209-24.

39. Shaham Y, Shalev U, Lu L, de Wit H, Stewart J. The reinstatement model of drug relapse: history, methodology and major findings. Psychopharmacology. 2003;168(1):3-20.

40. Koob GF, Volkow ND. Neurocircuitry of addiction. Neuropsychopharmacology. 2010;35(1):217-38

41. Etter JF, Bullen C. Electronic cigarette: users profile, utilization, satisfaction and perceived efficacy. Addiction (Abingdon, England). 2011;106(11):2017-28.

42. Koob GF, Volkow ND. Neurobiology of addiction: a neurocircuitry analysis. Lancet Psychiatry. 2016;3(8):760-73.
43. Liu G, Wasserman E, Kong L, Foulds J. A comparison of nicotine dependence among exclusive e-cigarette and cigarette users in the PATH study. Prev Med. 2017;104:86-91.

44. Rostron BL, Schroeder MJ, Ambrose BK. Dependence symptoms and cessation intentions among US adult daily cigarette, cigar, and e-cigarette users, 2012-2013. BMC Public Health. 2016;16(1):814.

45. Strong DR, Pearson J, Ehlke S, Kirchner T, Abrams D, Taylor K, Compton WM, Conway KP, Lambert E, Green VR, et al. indicators of dependence for different types of tobacco product users: descriptive findings from wave 1 (2013-2014) of the population assessment of tobacco and health (PATH) study. Drug Alcohol Depend. 2017;178:257-66.

46. Etter JF, Eissenberg T. Dependence levels in users of electronic cigarettes, nicotine gums and tobacco cigarettes. Drug Alcohol Depend. 2015;147:68-75.

47. Farsalinos KE, Romagna G, Tsiapras D, Kyrzopoulos S, Voudris V. Evaluating nicotine levels selection and patterns of electronic cigarette use in a group of "vapers" who had achieved complete substitution of smoking. Subst Abuse. 2013;7:139-46.

48. Steinberg MB, Zimmermann MH, Delnevo CD, Lewis MJ, Shukla P, Coups EJ, Foulds J. E-cigarette versus nicotine inhaler: comparing the perceptions and experiences of inhaled nicotine devices. J Gen Int Med. 2014;29(11):1444-50.

49. Stiles MF, Campbell LR, Graff DW, Jones BA, Fant RV, Henningfield JE. Pharmacodynamic and pharmacokinetic assessment of electronic cigarettes, combustible cigarettes, and nicotine gum: implications for abuse liability. Psychopharmacology. 2017;234(17):2643-55.

50. Vansickel AR, Weaver MF, Eissenberg T. Clinical laboratory assessment of the abuse liability of an electronic cigarette. Addiction. 2012;107(8):1493-500.

51. Pericot-Valverde I, Yoon JH, Gaalema DE. Single- and cross-commodity delay discounting of money and e-cigarette liquid in experienced e-cigarette users. Drug Alcohol Depend. 2020;206:107740.

52. Berridge $K C$, Robinson TE. Liking, wanting, and the incentive-sensitization theory of addiction. Am Psychol. 2016;71 (8):670-9.

53. Everitt BJ, Robbins TW. Neural systems of reinforcement for drug addiction: from actions to habits to compulsion. Nat Neurosci. 2005;8(11):1481-9.

54. Robinson TE, Berridge KC. Review. The incentive sensitization theory of addiction: some current issues. Philos Trans R Soc Lond Ser B Biol Sci. 2008;363(1507):3137-46.

55. McPherson S, Howell D, Lewis J, Barbosa-Leiker C, Bertotti Metoyer P, Roll J. Self-reported smoking effects and comparative value between cigarettes and high dose e-cigarettes in nicotine-dependent cigarette smokers. Behav Pharmacol. 2016;27(2 and 3-Special Issue):301-7.

56. Dowd AN, Tiffany ST. Comparison of tobacco and electronic cigarette reward value measured during a cue-reactivity task: an extension of the choice behavior under cued conditions procedure. Nicotine Tob Res Off J Soc Res Nicotine Tob. 2019;21(10):1394-400.

57. Bühler M, Vollstädt-Klein S, Kobiella A, Budde H, Reed LJ, Braus DF, Büchel C, Smolka MN. Nicotine dependence is characterized by disordered reward processing in a network driving motivation. Biol Psychiatry. 2010;67(8):745-52.

58. Chase HW, Eickhoff SB, Laird AR, Hogarth L. The neural basis of drug stimulus processing and craving: an activation likelihood estimation meta-analysis. Biol Psychiatry. 2011;70(8):785-93.

59. Engelmann JM, Versace F, Robinson JD, Minnix JA, Lam CY, Cui Y, Brown VL, Cinciripini PM. Neural substrates of smoking cue reactivity: a metaanalysis of fMRI studies. Neuroimage. 2012;60(1):252-62.

60. Kühn S, Gallinat J. Common biology of craving across legal and illegal drugs - a quantitative meta-analysis of cue-reactivity brain response. Eur J Neurosci. 2011;33(7):1318-26.

61. Lin X, Deng J, Shi L, Wang Q, Li P, Li H, Liu J, Que J, Chang S, Bao Y, et al. Neural substrates of smoking and reward cue reactivity in smokers: a meta-analysis of fMRI studies. Transl Psychiatry. 2020;10(1):97.

62. Nichols TT, Foulds J, Yingst JM, Veldheer S, Hrabovsky S, Richie J, Eissenberg T, Wilson SJ. Cue-reactivity in experienced electronic cigarette users: novel stimulus videos and a pilot fMRI study. Brain Res Bull. 2016:123:23-32.

63. Wall MB, Mentink A, Lyons G, Kowalczyk OS, Demetriou L, Newbould RD. Investigating the neural correlates of smoking: feasibility and results of combining electronic cigarettes with fMRI. Sci Rep. 2017;7(1):11352. 
64. Everitt BJ. Neural and psychological mechanisms underlying compulsive drug seeking habits and drug memories-indications for novel treatments of addiction. Eur J Neurosci. 2014;40(1):2163-82.

65. Field M, Cox WM. Attentional bias in addictive behaviors: a review of its development, causes, and consequences. Drug Alcohol Depend. 2008;97(1-2):1-20.

66. Kwak SM, Na DL, Kim G, Kim GS, Lee JH. Use of eye movement to measure smokers' attentional bias to smoking-related cues. Cyberpsychol Behav Impact Internet Multimed Virtual Real Behav Soc 2007; 10(2):299-304

67. Mogg K, Bradley BP, Field M, De Houwer J. Eye movements to smokingrelated pictures in smokers: relationship between attentional biases and implicit and explicit measures of stimulus valence. Addiction. 2003;98(6):825-36.

68. Mogg K, Field M, Bradley BP. Attentional and approach biases for smoking cues in smokers: an investigation of competing theoretical views of addiction. Psychopharmacology. 2005;180(2):333-41.

69. Vollstädt-Klein S, Loeber S, Winter S, Leménager T, von der Goltz C, Dinter C, Koopmann A, Wied C, Winterer G, Kiefer F. Attention shift towards smoking cues relates to severity of dependence, smoking behavior and breath carbon monoxide. Eur Addict Res. 2011;17(4):217-24.

70. Bradley B, Field M, Mogg K, De Houwer J. Attentional and evaluative biases for smoking cues in nicotine dependence: component processes of biases in visual orienting. Behav Pharmacol. 2004;15(1):29-36.

71. Bradley BP, Mogg K, Wright T, Field M. Attentional bias in drug dependence: vigilance for cigarette-related cues in smokers. Psychol Addict Behav. 2003;17(1):66-72

72. Lochbuehler K, Wileyto EP, Tang KZ, Mercincavage M, Cappella $J N$, Strasser AA. Do current and former cigarette smokers have an attentional bias for e-cigarette cues? J Psychopharmacol (Oxf Engl). 2018;32(3):316-23.

73. Faul F, Erdfelder E, Lang A-G, Buchner A. G*Power 3: a flexible statistical power analysis program for the social, behavioral, and biomedical sciences. Behav Res Methods. 2007;39(2):175-91.

74. Vollstädt-Klein S, Loeber S, Richter A, Kirsch M, Bach P, von der Goltz C, Hermann D, Mann K, Kiefer F. Validating incentive salience with functional magnetic resonance imaging: association between mesolimbic cue reactivity and attentional bias in alcohol-dependent patients. Addict Biol. 2012;17(4):807-16.

75. Christiansen P, Mansfield R, Duckworth J, Field M, Jones A. Internal reliability of the alcohol-related visual probe task is increased by utilising personalised stimuli and eye-tracking. Drug Alcohol Depend. 2015;155:170-4

76. Schmukle SC. Unreliability of the dot probe task. Eur J Personal. 2005;19(7):595-605

77. Beesdo-Baum K, Zaudig M, Wittchen H-U, editors. SCID-5-CV: strukturiertes klinisches Interview für DSM-5-Störungen-Klinische Version: deutsche Bearbeitung des structured clinical interview for DSM-5 disorders_clinician version von Michael B. First, Janet B.W. Williams, Rhonda S. Karg, Robert L. Spitzer, 1, Auflage edn. Göttingen: Hogrefe; 2019.

78. Scheurich A, Müller MJ, Anghelescu I, Lörch B, Dreher M, Hautzinger M, Szegedi A. Reliability and validity of the form 90 interview. Eur Addict Res. 2005;11(1):50-6.

79. Heatherton TF, Kozlowski LT, Frecker RC, Fagerstrom K-O. The Fagerström test for nicotine dependence: a revision of the Fagerstrom tolerance questionnaire. Br J Addict. 1991;86:9S.

80. Vollstädt-Klein S, Leménager T, Jorde A, Kiefer F, Nakovics H. Development and validation of the craving automated scale for alcohol. Alcoho Clin Exp Res. 2015;39(2):333-42.

81. Cox LS, Tiffany ST, Christen AG. Evaluation of the brief questionnaire of smoking urges (QSU-brief) in laboratory and clinical settings. Nicotine Tob Res. 2001;3:7-16.

82. Dowd AN, Motschman CA, Tiffany ST. Development and validation of the questionnaire of vaping craving. Nicotine Tob Res. 2019;21:63-70.

83. Rash C, Copeland A. The brief smoking consequences questionnaireadult (BSCQ-A): development of a short form of the SCQ-A. Nicotine Tob Res. 2008;10:1633-43.
84. Hughes J. Effects of abstinence from tobacco: valid symptoms and time course. Nicotine Tob Res. 2007;9:315-27.

85. Cohen S, Kamarck T, Mermelstein R. A global measure of perceived stress. J Health Soc Behav. 1983;24(4):385-96.

86. Crawford JR, Henry JD. The positive and negative affect schedule (PANAS): construct validity, measurement properties and normative data in a large non-clinical sample. Br J Clin Psychol. 2004;43:21S.

87. Preuss UW, Rujescu D, Giegling I, Watzke S, Koller G, Zetzsche T, Meisenzahl EM, Soyka M, Möller HJ. Psychometrische evaluation der deutschsprachigen version der Barratt-Impulsiveness-Skala psychometric evalutation of the German version of the Barratt Impulsiveness Scale. Nervenarzt. 2008;79:305-19.

88. Greenwald AG, McGhee DE, Schwartz JLK. Measuring individual differences in implicit cognition: the implicit association test. J Personal Soc Psychol. 1998;74:1464-80

89. Kirby KN, Maraković NN. Delay-discounting probabilistic rewards: rates decrease as amounts increase. Psychon Bull Rev. 1996;3(1):100-4.

90. Bechara A, Tranel D, Damasio H. Characterization of the decision-making deficit of patients with ventromedial prefrontal cortex lesions. Brain (London, England: 1878). 2000;123:2189-202.

91. Schulz M, Mack B, Renn O, editors. Fokusgruppen in der empirischen Sozialwissenschaft: Von der Konzeption bis zur Auswertung. Wiesbaden: VS Verl. für Sozialwiss; 2012.

92. Mayring P: Qualitative Inhaltsanalyse : Grundlagen und Techniken, 11. aktualisierte und überarb. Aufl. edn. Weinheim [u.a.]: Beltz; 2010.

93. Colloseus C. Grenzen erfahren—von Grenzen erzählen. Die Hebamme. 2016;29:33-8

94. Karlheim C. Depressions-online-foren: Präventiver Nutzen oder ein Risiko für Betroffene und Angehörige? In: Hahn S, Harald S, Abderhalden C, Needham I, Schulz M, Hegedüs A, Schoppmann S, editors. Gesundheitsförderung und Gesundheitskompetenz": eine Herausforderung für die psychiatrische Pflege in Praxis, Management, Ausbildung Forschung: Vorträge, Workshops und Posterpräsentationen 9 Dreiländerkongress Pflege in der Psychiatrie in Wien. Bern: Abt. Forschung/Entwicklung Pflege und Pädagogik, Universitäre Psychiatrische Dienste Bern; 2012. p. 166-7.

95. Kahr MK, Padgett S, Shope CD, Griffin EN, Xie SS, Gonzalez PJ, Levison J, Mastrobattista J, Abramovici AR, Northrup TF, et al. A qualitative assessment of the perceived risks of electronic cigarette and hookah use in pregnancy. BMC Public Health. 2015;15:1273.

96. Roberts LD. Ethical issues in conducting qualitative research in online communities. Qual Res Psychol. 2015;12:314-25.

97. Thompson ME, Fong GT, Hammond D, Boudreau C, Driezen P, Hyland A, Borland R, Cummings KM, Hastings GB, Siahpush M, et al. Methods of the international tobacco control (ITC) four country survey. Tob Control. 2006;15(Suppl 3):iii12-18.

98. Fong GT, Cummings KM, Borland R, Hastings G, Hyland A, Giovino GA Hammond D, Thompson ME. The conceptual framework of the international tobacco control (ITC) policy evaluation project. Tob Control. 2006;15(Suppl 3):iii3-11.

99. Vardavas $\mathrm{Cl}$, Bécuwe N, Demjén T, Fernández E, McNeill A, Mons U, Tountas Y, Trofor AC, Tsatsakis A, Rohde G, et al. Study protocol of European regulatory science on tobacco (EUREST-PLUS): policy implementation to reduce lung disease. Tob Induc Dis. 2018;16:A2.

100. Fong GT, Thompson ME, Boudreau C, Bécuwe N, Driezen P, Agar TK, Quah ACK, Zatoński WA, Przewoźniak K, Mons U, et al. The conceptual model and methods of wave 1 (2016) of the EUREST-PLUS ITC 6 European countries survey. Tob Induc Dis. 2018;16:A3.

101. Thompson ME, Driezen P, Boudreau C, Bécuwe N, Agar TK, Quah ACK, Zatoński W, Przewoźniak K, Mons U, Demjén T, et al. Methods of the international tobacco control (ITC) EUREST-PLUS ITC Europe surveys. Eur J Public Health. 2020;30(Suppl_3):4-9.

\section{Publisher's Note}

Springer Nature remains neutral with regard to jurisdictional claims in published maps and institutional affiliations. 